\title{
Application of hydrocyclone technology to tailings storage facilities to reduce water consumption
}

\author{
M.A. Knight Fraser Alexander Tailings, South Africa
}

J.A. Wates Fraser Alexander Tailings, South Africa

I. Du Plessis Multotec, South Africa; and Hatch, South Africa

\section{Abstract}

Cycloning technology is extensively used in mineral processing plants for the extraction and size separation of minerals. Cycloning of tailings storage facilities (TSFs) has been utilised successfully in South Africa as an alternative to other tailings deposition methods.

In order to evaluate paste and thickened tailings disposal options against alternative methods, one should make the comparison against realistic baseline water consumption. This may require optimisation of existing operations or, alternatively, preparation of an appropriate base case that has been designed to reduce water consumption to the minimum level possible without thickening. Cycloning or classification of tailings provides an opportunity to reduce water consumption in some cases.

Based on a number of actual case studies, Fraser Alexander and Multotec have worked together to develop a scientific approach for the design and operation of cyclone TSFs. The study focuses on taking cyclone practice from the mineral processing environment and combining it with TSF design thinking to optimise the design and operation of TSFs in such a manner as to optimise classified tailings production and to reduce water consumption as far as possible.

The approach is based on theoretical assessment, laboratory test work, field tests and observations and operational experience in managing TSFs.

The approach consists of the following steps:

- Evaluation of input parameters such as material properties, pressure and slurry relative density (RD).

- Use of a computer simulation package to provide the solids and water balance, and the particle size distributions of the product streams.

- Determination of the geotechnical properties of the underflow and overflow from samples generated by laboratory test work.

- Utilisation of output parameters to prepare a concept design for the TSF.

The paper will use a case study to illustrate the influence of variables such as rate of rise, cyclone split, and feed slurry solids concentration on the design of aspects of cyclone TSFs and on performance with respect to water consumption and recovery.

\section{$1 \quad$ Introduction}

The hydrocyclone was patented in 1912 and has subsequently been widely used in the minerals processing industry as a classifier for size separation in the mineral extraction process. The reasons for its wide spread use has been its ease of operation, high capacity and efficiency. A cyclone is a specialised device which separates particles by creating centrifugal forces in the slurry stream. Although a cyclone may be relatively 
easy to operate, the understanding of the numerous aspects influencing optimal performance is complex (Wills and Napier-Munn, 2007).

The efficiency of a cyclone in size separation lends itself to the effective application in the construction of TSFs. There are a number of configurations of the application of cyclones to construction of TSFs, but the most widely used one in South Africa is on-wall cycloning. The advantage of using cyclones in the construction of a TSF is the ability to separate the feed tailings stream into coarse and fine fractions, the cyclone underflow and overflow respectively which allows for building of coarse outer walls of the TSF.

By combining cyclone theory with the design aspects of TSFs, optimisation of the performance of the cyclone to produce the required quantity and quality of classified tailings can be achieved. This paper focuses on the application of existing cyclone theory from the mineral processing environment and combining it with TSF design thinking to optimise classified tailings production.

With overall water consumption becoming an important factor in a TSF operation, the comparison of various deposition methods to baseline water consumption is important. This paper discusses various aspects relating to water consumptions of cyclone TSFs and a case study to illustrate these is presented.

\section{Background}

The performance of the hydrocyclone is affected by a number of input parameters such as feed solids concentration (usually measured as slurry RD), particle size, particle density and feed pressure. Added to this, the underflow requirements for the construction of a TSF outer wall such as grading, permeability, stacking of underflow and percentage split between underflow and overflow, introduces a number of additional variables into the design of hydrocyclones for a specific TSF.

A key requirement for the design and operation of a cyclone TSF is to be able to accurately predict the cyclone split, as well as the properties of the respective underflow and overflow material. To achieve this, a process has been developed that ties the theory of hydrocyclones to the design and operational aspects critical for the successful construction of a cyclone TSF.

\section{Theory of hydrocyclones}

The theory of hydrocyclones has been well developed in the mineral processing environment. There are numerous factors that influence cyclone performance in their application for the construction of TSFs such as:

- The feed characteristic of material fed to the cyclone, which includes, but is not limited to, $\%$ solids, particle size distribution, and mineralogy of the solids.

- The cyclone configuration which has numerous permutations including cyclone size, vortex finder diameter, spigot size, cyclone feed pressure and cone angle.

The outcome of the evaluation of the cyclone theory is being able to determine the relationships between the various input parameters, some of which can be altered and some which cannot, to optimise performance of cyclones for building TSFs. Table 1 summarises the relationship between various parameters on the performance of the cyclones in relation to their use for building TSFs. 
Table 1 Summary of the influence of cyclone inputs on the cyclone performance

\begin{tabular}{clc}
\hline $\begin{array}{c}\text { Direction of Change } \\
\text { of Input Parameter }\end{array}$ & Input Parameter & $\begin{array}{c}\text { Influence on } \\
\text { Cyclone Cut Size }\end{array}$ \\
\hline$\uparrow$ & Cyclone feed RD & $\uparrow$ \\
$\uparrow$ & Particle density & $\downarrow$ \\
$\uparrow$ & Feed pressure & $\downarrow$ \\
$\uparrow$ & Vortex finder & $\uparrow$ \\
$\uparrow$ & Spigot/vortex finder ratio & $\downarrow$ \\
$\uparrow$ & Cyclone diameter & $\uparrow$ \\
\hline
\end{tabular}

\section{4}

Cyclone selection for TSF

In the application of cycloning to construction of TSFs, the most critical factor is being able to accurately determine cyclone performance at the design stage. In order to achieve this, theoretical assessment, laboratory test work, field tests and observations to calibrate cyclone performance based on input parameters and required output criteria are carried out to optimise cyclone performance.

\section{1 Computer simulation and calibration of cyclones}

Proprietary software which encompasses the theory of cycloning and the factors influencing cyclone performance (Plitt, 1976) can be used to specify cyclones for the construction of TSFs. Input data such as feed tonnages per hour, RD, pressure, particle size distribution and specific gravity (SG), as listed in section 3 , are entered into the computer simulation. The output of the software is generated to provide information on the \% split achieved and suitable cyclone sizing. The outputs are compared against requirements for the TSF such as \% split required to underflow, pressure available and practical considerations such as number of cyclones required.

\section{2 Laboratory and field tests}

Once the simulation has shown that the range of parameters is within acceptable limits, a sample of the feed material is sent for laboratory testing. This is run through a cyclone test rig (see Figure 1) to calibrate the cyclone specified and generate samples of the underflow to allow for testing of its geotechnical properties.
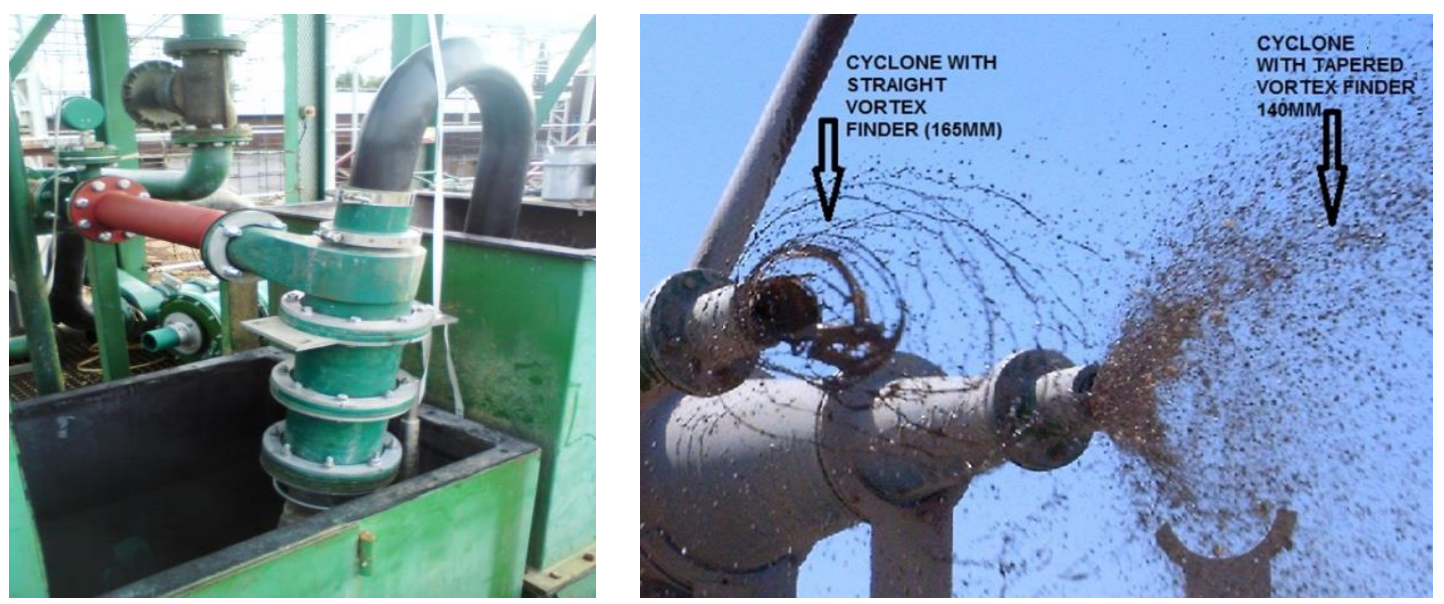

Figure 1 Cyclone test rigs (laboratory and site) 
In some instances, field testing is conducted on existing TSFs to verify the laboratory results for actual site conditions. This is only possible if there is an existing TSF operating and the cyclone investigation is for a new TSF or conversion from another deposition method to cycloning.

In both the laboratory and field testing, actual samples of the underflow and overflow are generated and these can then be sent to a soils laboratory to test the engineering properties of the material such as particle size distribution (PSD), friction angle and permeability.

The advantage of both the laboratory and onsite testing is that a number of tests can be run varying input parameters to achieve optimal cyclone performance. This provides invaluable information on the sensitivity of parameters in the design and construction of the TSF.

\section{Application of cyclone theory to TSFs}

There are a number of configurations in which cyclones are used in the construction of TSFs. The common variants range from cyclone plants used off the dam that produce sand which is then pumped onto the perimeter of the TSF, to 'on-wall' cyclones that place the underflow directly on the outer wall. In South Africa, the most widely used application is 'on-wall' cycloning (Robinson, 2010). Figure 2 shows a typical cross-section for a cycloned TSF.

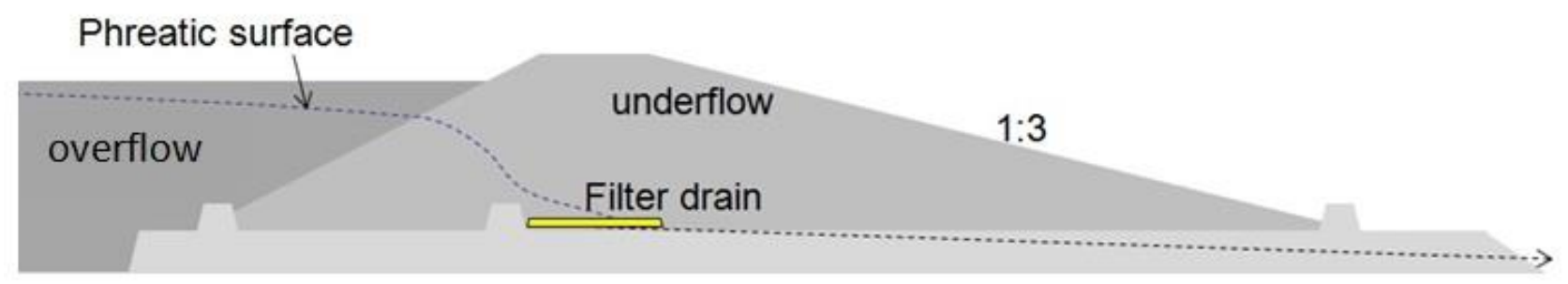

Figure 2 Typical section of cyclone TSF

Figure 3 shows a typical on wall cyclone operations on a platinum TSF. Note that for ease of use the cyclone has been placed on a wheeled stand.
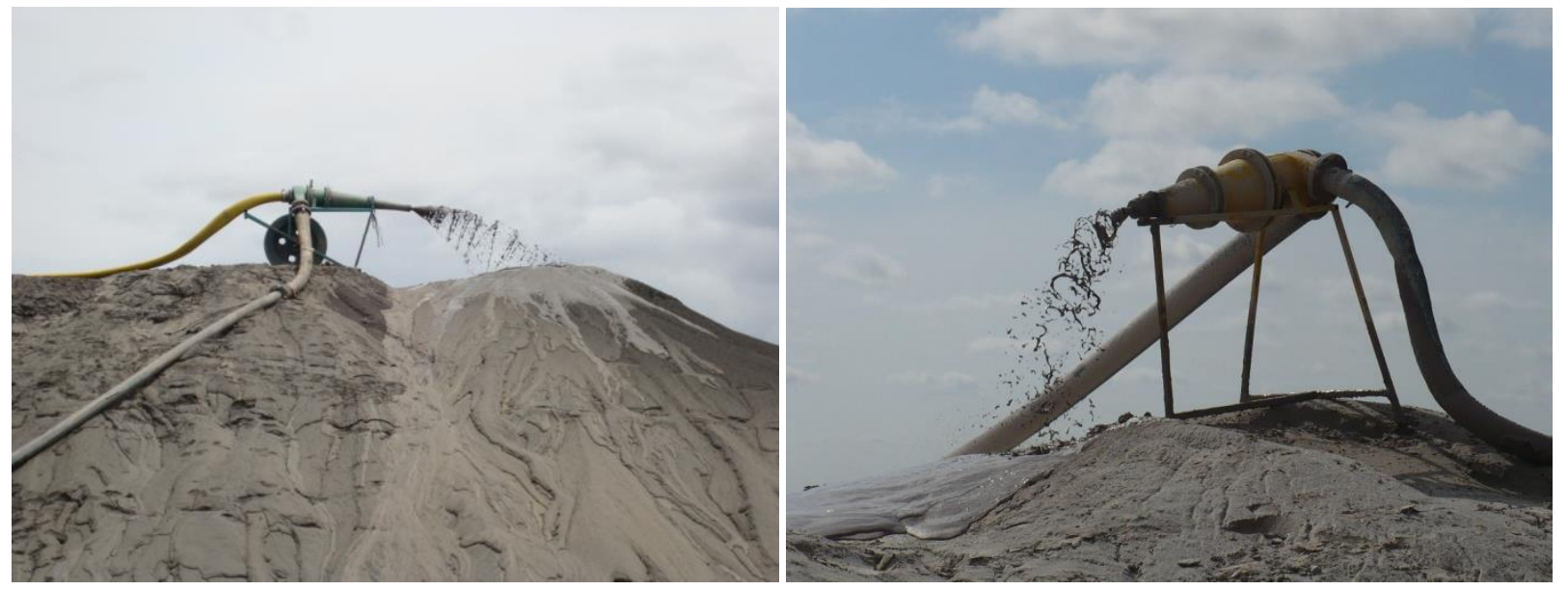

Figure 3 0n-wall cyclone on TSF (platinum and gold)

The key criteria when using cyclones to construct a TSF is the quantity and quality of the underflow. Through the test work and application of the theory as described in sections 3 and 4, the quality of the underflow material can be determined at design stage to check if it meets the following criteria:

- The underflow has a sufficiently steep angle of repose to enable wall building.

- The \% mass and volume split is sufficient to meet the geometric requirements for the TSF design. 
- The permeability of the underflow is acceptable to provide for an adequate contrast with the overflow permeability and to allow free draining.

- The shear strength development is sufficient based on the rate of rise.

- The drying or desaturation rate is fast enough to permit access in time to move cyclones.

All of these criteria are crucial to the safe and efficient functioning of the TSF throughout its design life and by evaluating these at concept stage, a high level of confidence can be provided in the design.

Fraser Alexander and Multotec have been working together to develop a tracked cyclone unit which is shown in Figure 4. A number of these tracked cyclones have been deployed on the MWS Kareerand TSF where the deposition is in the region of 1.7-2.0 million tonnes per month (tpm). An aerial view of this TSF is also shown in Figure 4.
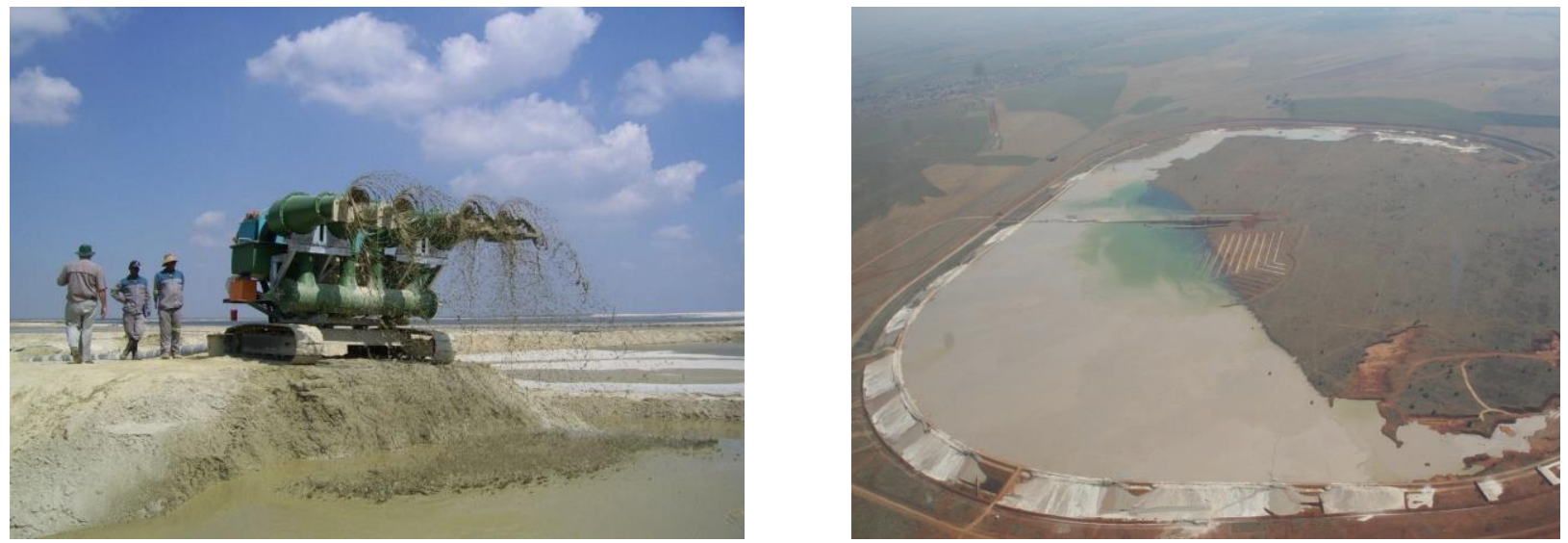

Figure 4 Cyclone TSF built with tracked cyclone units

\section{$6 \quad$ Water consumption of cyclone TSF}

The system used for the disposal of tailings is the main contributor to the water consumed for mining as firstly, based on the slurry density, it determines the amount of water sent to the tailings deposition site. Secondly the amount of water 'lost' at the deposition site is determined by a number of factors such as: seepage, evaporation and water locked up in the tailings. In addition, the evaporation losses at the return water dam and in the return water system can result in lower water recoveries from the TSF.

Cycloning or classification of tailings can, in some cases, result in increased water savings when compared to the baseline water consumption of various deposition methods, based on the following reasons:

- Higher rate of rise allows for deposition of greater tonnages on a smaller footprint. Having a smaller footprint results in a reduced area for evaporation and seepage from the TSF.

- On a multi-deposition point cyclone dam, the beach remains wet (refer Figure 5), which results in more water reporting to the decant system.

- Due to the higher permeability of the outer walls in a cyclone TSF, an improved water recovery can be achieved from a well-designed underdrain system.

Effective operations of a cyclone TSF can result in high water recoveries and this may be a preferred deposition option in some cases based on cost and ease of implementation. The case study presented in section 7 illustrates the operational aspects as well as the water balance for a cycloned gold TSF.

\section{$7 \quad$ Case study - cyclone TSF (MWS dam 5 gold tailings)}

The case study presented here is a cycloned gold TSF built using on-wall $250 \mathrm{~mm}$ diameter cyclones. The tailings are from a hydraulic re-mining operation. 


\section{1 Overview of TSF}

The TSF was converted from a normal daywall operation to a cyclone TSF in order to accommodate a higher rate of rise as a result of the high tonnages being placed on a relatively small footprint. A crucial factor in the success of this TSF was the cyclone performance, especially given the fine grading of the feed material. This resulted in significant challenges in achieving the required split and effective stacking of the underflow in order to build the outer wall.

This TSF was utilised from January 2008 until March 2011, at which time the deposition was diverted to the MWS Kareerand TSF built with track mounted cyclones referred to in section 5.

The key details relating to the TSF are summarised:

- Deposition of 1.2 million tpm, two streams of 0.6 million tpm.

- Feed slurry density varied between 1.3 and 1.4.

- The target split to underflow required to achieve the TSF geometry was $14 \%$.

- Average rate of rise of TSF is $9.0 \mathrm{~m}$ per year.

- The dam was initially built downstream and then converted to upstreaming towards the end of life.

Figure 5 shows an aerial view of the TSF as well as view along the underflow wall being built with the onwall cycloning method.
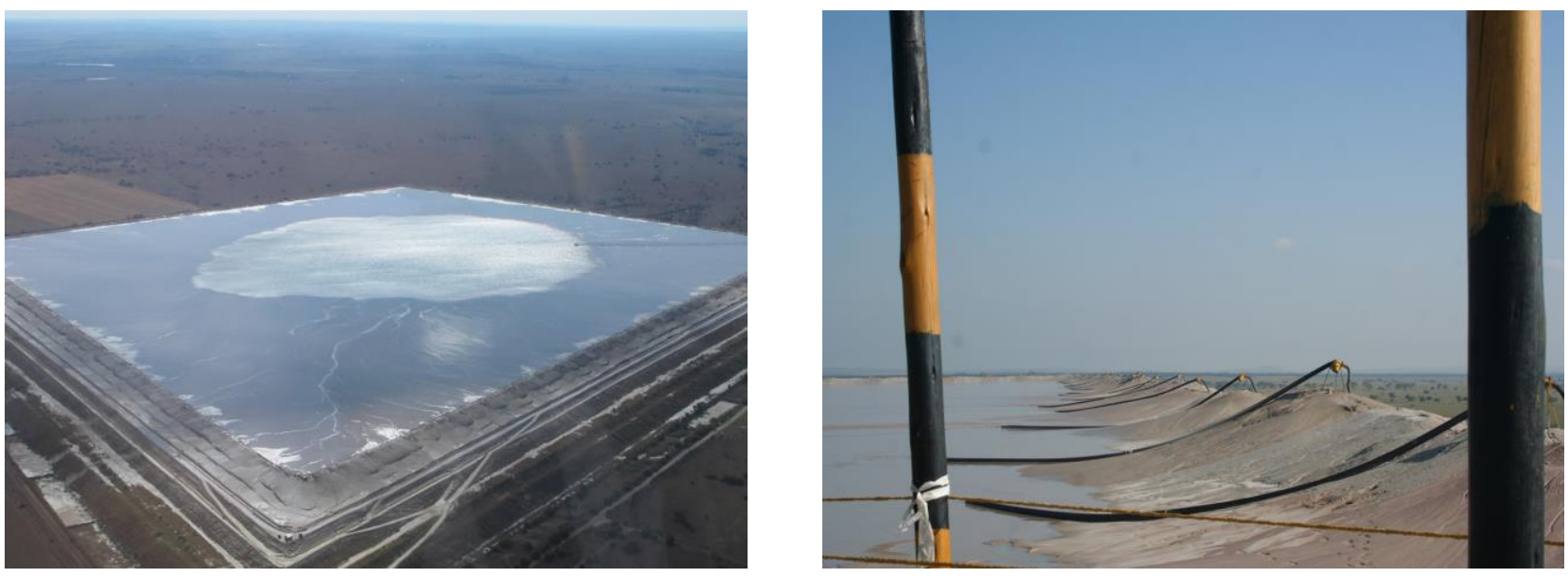

Figure 5 MWS dam 5 cycloned TSF

Due to the high rate of rise, extensive management controls were put in place to ensure the optimal development of the TSF. This included detailed reporting on key aspects such as percentage split achieved, feed RD, drain flows, piezometer readings and DCP test results. In addition, detailed bi-monthly assessments were done to assess volumetric split measured by survey, stability and remaining life (Davel, 2011).

The extensive monitoring carried out during the life of the TSF resulted in accurate recording of all operational and technical data, including a detailed water balance carried out by the personnel on the mine.

\section{2 Water balance results}

A detailed water balance was carried out on this TSF over a period of seven months including the remining, gold plant and tailings dam. A typical monthly water balance is shown in Figure 6. 
TYPICAL MONTHLY WATER BALANCE

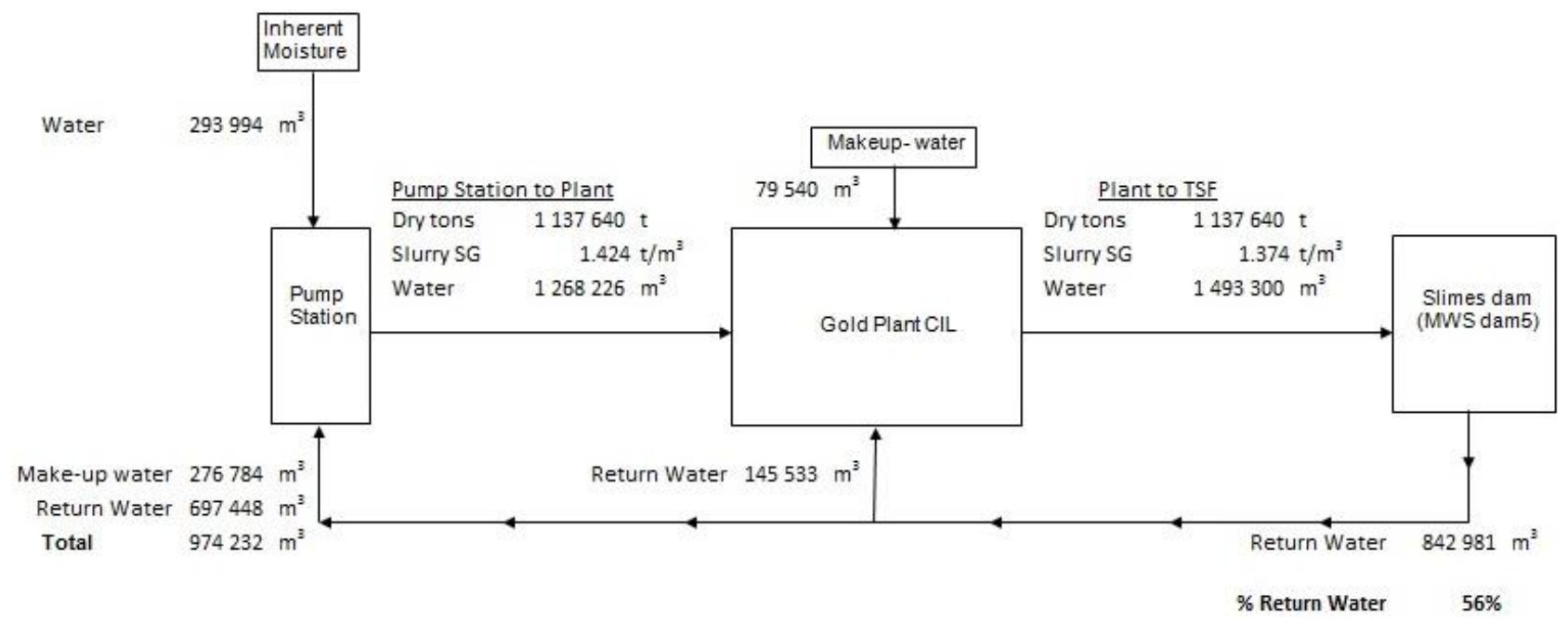

Figure 6 Typical monthly water balance

The total water recovered from the TSF during this period calculated as a ratio of the feed to return water is:

Water recovery $=$ Return Water $/$ Feed water $=49 \%$. The monthly variances ranged from $44-56 \%$.

The amount of water used over the period was $5,414 \times 10^{3} \mathrm{~m}^{3}$ for an equivalent $8,082 \times 10^{3}$ tonnes. This equates to a water consumption of $0.67 \mathrm{~m}^{3}$ per tonne milled for a feed RD of 1.38 . This is considerably less than the consumption reported of $0.80 \mathrm{~m}^{3}$ and $1.20 \mathrm{~m}^{3}$ (Wates, 1983) for conventional paddocked facilities at the adjacent Vaal Reefs Mine for slurry RDs of 1.45 and 1.25 respectively. These measured consumption rates are shown in Figure 7, which illustrates a generic water balance over a range of solids concentrations from dilute slurry to paste for gold tailings in the climatic region.

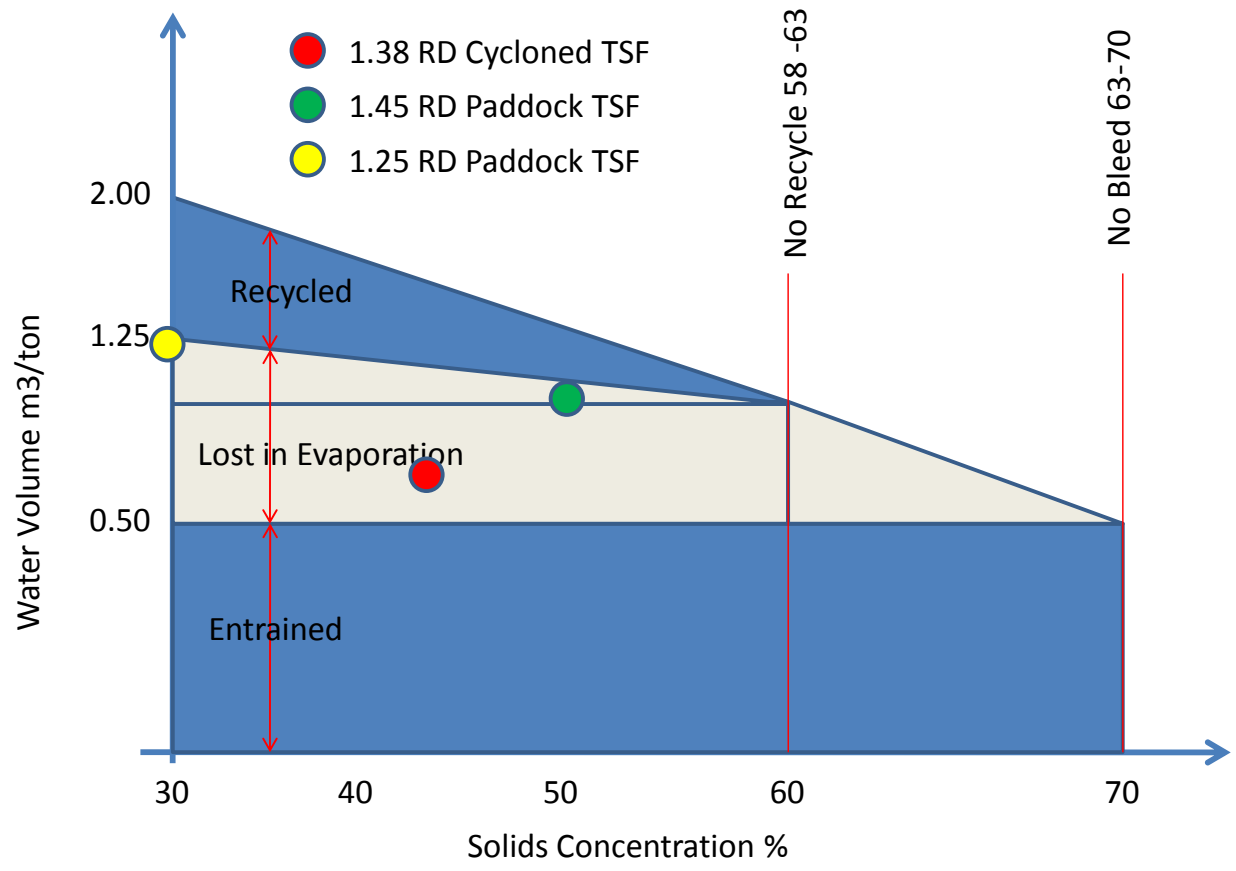

Figure 7 Generic water balance - range of solids concentrations 
Figure 7 shows that a paste conversion would be far more attractive when compared to the consumption that was measured for a $1.25 \mathrm{RD}$ paddocked facility with a possible saving of $0.70 \mathrm{~m}^{3}$ per tonne, than it would be when compared to the cyclone facility with only a possible saving of $0.17 \mathrm{~m}^{3}$ per tonne.

The graphical representation of the water balance over the period for the cyclone gold TSF in the case study is shown in Figure 8.

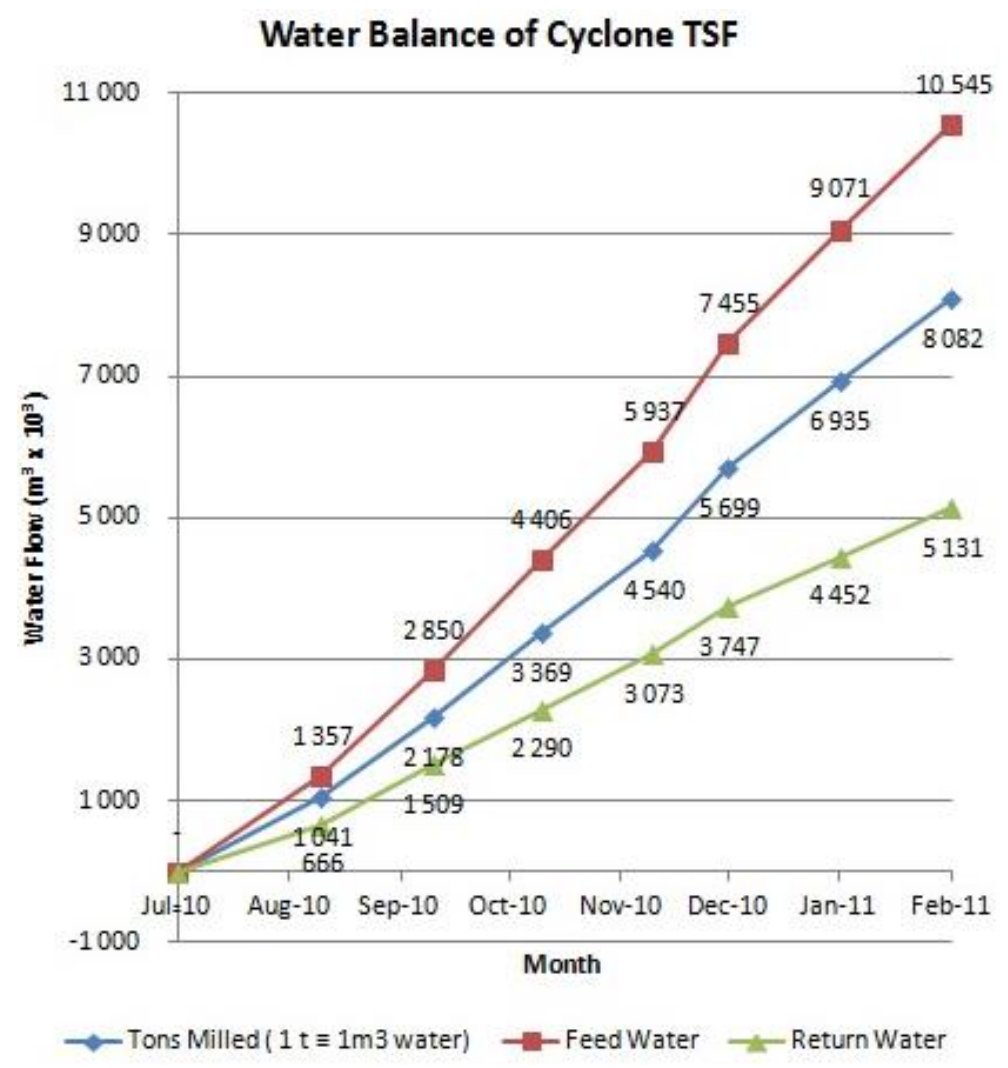

Figure 8 Water balance - cyclone gold TSF

Note that detailed information on evaporation and precipitation was not recorded and hence the results shown are the total water balance for the TSF inclusive of these factors. The evaporation on the return water dam was not quantified, but this loss is included in the total water recovery number.

In the case of gold tailings dams, the case study shows a higher water recovery than the conventional paddocked system.

\section{Conclusions}

Cycloning of TSFs provides a number of advantages as a deposition method that can also lead to reduction in water consumption. By applying hydrocyclone theory from the mineral processing environment to the design and operations of cyclone TSFs, a process has been developed that can optimise cyclone performance for TSF applications.

The process utilises cyclone simulation, laboratory and field tests work and takes into account the required output parameters of the cyclone underflow to ensure the design and operational criteria for the TSF can be met.

This means that during the conceptual design stage the risk of not meeting the performance criteria is evaluated and mitigated, which results in a significantly higher success rate of cycloning TSFs. This process has been utilised in the implementation of a unique tracked cyclone for building a high capacity TSF. 
The case study presented illustrated the application of cyclones to operating a TSF under extremely challenging conditions at a rate of rise of $9.0 \mathrm{~m}$ per annum. Through effective monitoring of the cyclone and TSF performance criteria such as, feed RD, pressure, split, DCP results and detailed bi-monthly stability and capacity assessment, the risk associated with the design and operation of the TSF were successfully managed.

The water balance conducted on this TSF shows a total water recovery of $49 \%$ which for the case of gold tailings dams is significantly higher than that for the conventional daywall method in the same climatic region where the range is closer to $25 \%$. In order to understand the individual components that make up the total water balance, further work is required to do a detailed water balance taking into account factors such as evaporation, seepage, drain flows and interstitial water.

\section{Acknow ledgements}

The authors would like to acknowledge the assistance provided by the personnel from Mine Waste Solutions (MWS) and Fraser Alexander Tailings for sourcing field data for this paper. A special word of appreciation to E. Mokoteli of MWS for providing the water balance data, as well as K. Van Staden and L. Tshonweni of Fraser Alexander for providing historical data for MWS Dam 5. Reviews of the paper by A. O'Callaghan and Prof. G.E. Blight were greatly appreciated.

\section{References}

Davel, K. (2011) Mine Waste Solution Bi-monthly Reports, created for First Uranium, by K. Davel, Johannesburg (confidential, cited with permission).

Plitt, L.R. (1976) A mathematical model of a hydrocyclone classifier, CIM Bulletin, December 1976, Vol. 69 (776), pp. 114-123.

Robinson, B. (2010) The fundamentals of on-wall cyclone tailings dams, presented at Tailings Disposal Course on behalf of Fraser Alexander, October 2010.

Wates, J.A. (1983) The Disposal of Mine tailings: The Real Cost of Excess Water in Residues, Journal of the South African Institute of Mining and Metallurgy, November/December 1983.

Wills, B.A. and Napier-Munn, T.J. (2007) Mineral Processing Technology, Butterworth-Heinermann, pp. 212-223. 
